Orthopäde 2015 - 44:566-573

DOI 10.1007/s00132-015-3115-y

Online publiziert: 6. Mai 2015

(c) Die Autor(en) 2015. Dieser Artikel ist auf

Springerlink.com mit Open Access verfügbar
K.P. Kutzner ${ }^{1}$ C. Paulini ${ }^{1} \cdot$ M. Hechtner ${ }^{2}$ P. Rehbein ${ }^{1} \cdot$ J. Pfeil ${ }^{1}$

${ }^{1}$ Klinik für Orthopädie und Unfallchirurgie, St. Josefs Hospital Wiesbaden, Wiesbaden, Deutschland

${ }^{2}$ Institut für medizinische Biometrie, Epidemiologie und Informatik,

Johannes Gutenberg Universität Mainz, Mainz, Deutschland

\title{
Postoperative Analgesie nach Knie-TEP
}

\section{Intraartikulärer Katheter vs. Nervus-femoralis-Katheter}

Die Implantation einer Knietotalendoprothese (Knie-TEP) ist ein weltweit etabliertes Standardverfahren zur Behandlung einer fortgeschrittenen Pangonarthrose und sorgt in vielen Fällen für einen deutlichen Anstieg der Lebensqualität. Deutliche postoperative Schmerzen führen jedoch bis zum heutigen Tage nicht selten zu Funktionseinschränkungen mit Verzögerung der Rehabilitation und tragen zu einer geminderten Zufriedenheit der Patienten bei. In diesem Beitrag werden in einem prospektiv randomisierten Vergleich zwei Techniken zur postoperativen Schmerztherapie vorgestellt und evaluiert.

\section{Hintergrund und Fragestellung}

Nach Implantation einer Knie-TEP ist das Auftreten einer deutlichen postoperativen Schmerzsymptomatik häufig. Die höchste Schmerzintensität wird in der Regel nach 3-6 $\mathrm{h}$ postoperativ erreicht und hält oft in den ersten Tagen an $[2,3]$. Das postoperative Schmerzmanagement, besonders in den ersten Tagen, stellt daher eine groBe Herausforderung dar, sowohl für den Patienten als auch für den behandelnden Arzt. Eine suffiziente postoperative Schmerztherapie führt nachweislich zu einer beschleunigten Funktionsverbesserung, einem verkürzten Krankenhausaufenthalt und einer größeren Patientenzufriedenheit $[4,5,9,26]$. Das Schmerzmanagement, vor allem in der frühen postoperativen Phase, scheint weiterhin groBe Bedeutung zu haben in Hinblick auf eine schnellere Mobilisation und Rehabilitation und im Hinblick auf die Reduzierung von Komplikationen [1, 7, 8, 22].

Bereits seit einigen Jahren finden regelhaft regionale Anästhesieverfahren zur Unterstützung der oralen Medikation Anwendung. Eine systemische Therapie mittels nichtsteroidaler Antirheumatika (NSAR) und Opioiden ist mit bekannten Nebenwirkungen wie Nausea, Erbrechen, Kreislaufschwankungen und Obstipation vergesellschaftet, welche sich dramatisch auf den Patientenkomfort und die stationäre Aufenthaltsdauer auswirken können $[11,23]$.Zusätzliche Maßnahmen sollen vor allem den systemischen Opioidbedarf senken. Besonders der Nervus-femoralis-Katheter (NFK) ist in vielen Kliniken weit verbreitet. Viele Studien konnten die
Wirksamkeit des NFK zeigen [11, 19]. Jedoch gibt es auch Nachteile: So deckt das Versorgungsgebiet des N. femoralis den dorsalen Anteil inklusive Kniekehle nicht ab. Außerdem besteht aufgrund motorischer Blockade und Sensibilitätsverlust im Bereich der unteren Extremität eine erhöhte Sturzgefahr. Folglich kommt es $\mathrm{zu}$ einer verzögerten postoperativen Mobilisation [12, 21]. Zusätzlich ist die Applikation des Katheters technisch nicht immer möglich und bedarf nicht selten eines deutlichen zeitlichen Aufwandes im Rahmen der Operationsvorbereitung.

In den letzten Jahren sind vor allem im Rahmen von Fast-track-Konzepten verschiedene multimodale Konzepte zur Schmerzbehandlung vorgestellt worden $[15,17,18]$. Sie enthalten unterschiedli-

\begin{tabular}{|c|c|c|c|c|c|c|}
\hline & IAK & & NFK & & Gesamt & \\
\hline & $n(\%)$ & $M W \pm S D$ & $n(\%)$ & $M W \pm S D$ & $n(\%)$ & $M W \pm S D$ \\
\hline \multicolumn{7}{|l|}{ Geschlecht } \\
\hline Männlich & $20(33,3)$ & & $23(38,3)$ & & $43(35,8)$ & \\
\hline Weiblich & $40(66,7)$ & & $37(61,7)$ & & $77(64,2)$ & \\
\hline Alter & & $70,5 \pm 8,0$ & & $70,6 \pm 8,6$ & & $70,5 \pm 8,3$ \\
\hline \multicolumn{7}{|c|}{ Operierte Seite } \\
\hline Rechts & $39(65,0)$ & & $31(51,7)$ & & $70(58,3)$ & \\
\hline Links & $21(35,0)$ & & $29(48,3)$ & & $50(41,7)$ & \\
\hline $\begin{array}{l}\text { Einleitungs- } \\
\text { zeit [min] }\end{array}$ & & $42,8 \pm 10,0$ & & $54,7 \pm 9,8$ & & $48,7 \pm 11,5$ \\
\hline $\begin{array}{l}\text { Schnitt-Naht- } \\
\text { Zeit [min] }\end{array}$ & & $75,3 \pm 21,1$ & & $81,0 \pm 23,9$ & & $78,2 \pm 22,6$ \\
\hline Stationäre & & $8,6 \pm 2,2$ & & $9,0 \pm 2,2$ & & $8,8 \pm 2,2$ \\
\hline $\begin{array}{l}\text { Aufenthalts- } \\
\text { dauer [Tage] }\end{array}$ & & $8,0(7,0-10,0)^{a}$ & & $8,0(7,0-10,0)^{a}$ & & $8,0(7,0-10,0)^{a}$ \\
\hline
\end{tabular}




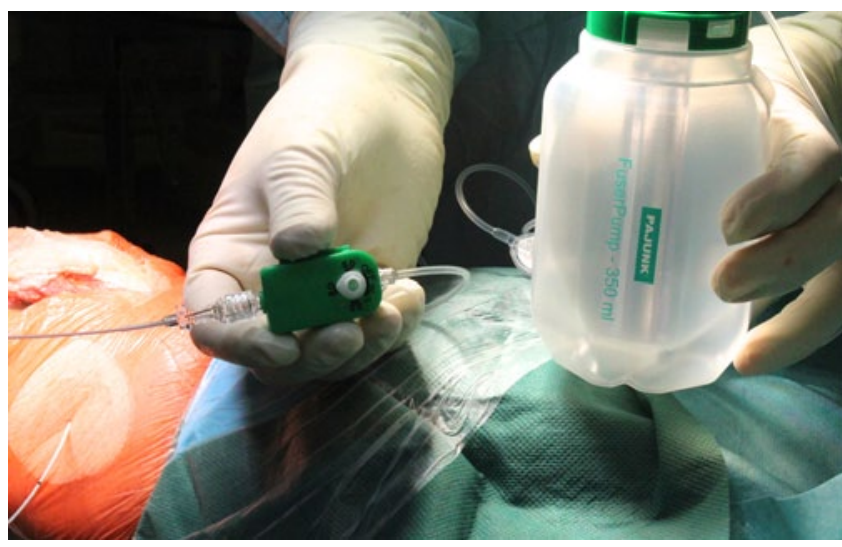

Abb. 1 A Intraoperative Applikation eines intraartikulären Katheters mit Elastomerpumpe (InfiltraLong, FuserPump; Fa. Pajunk ${ }^{\circledR}$ )

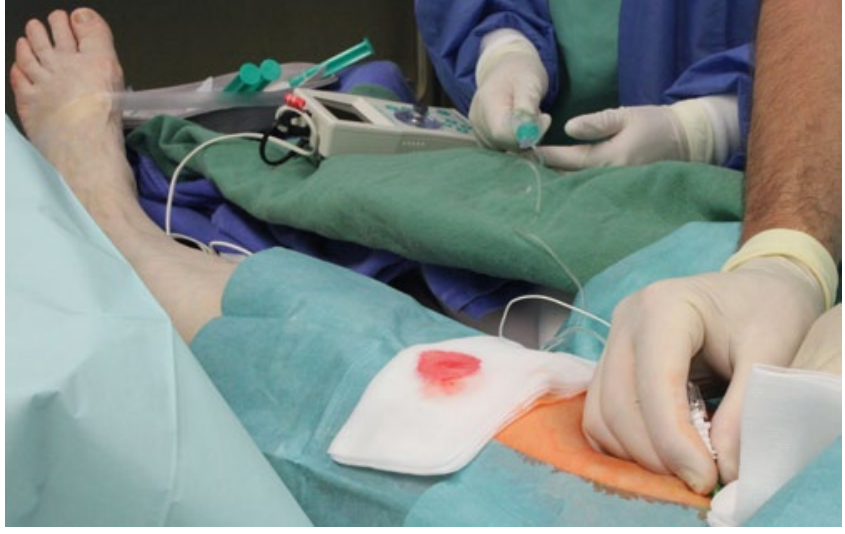

Abb. 2 A Präoperative Applikation eines Nervus-femoralis-Katheters (PlexoLong/Meier; Fa. Pajunk ${ }^{\circledR}$ ) che Kombinationen von Techniken und Medikamenten, um neben einer effizienten Schmerztherapie auch eine zügige Mobilisation und eine Reduzierung von Nebenwirkungen zu ermöglichen. Ein wichtiger Bestandteil vieler dieser Konzepte ist die intraoperative periartikuläre Infiltrationsbehandlung mittels Lokalanästhetika. Eine gute Wirksamkeit konnte in einigen Studien nachgewiesen werden $[6,17,18]$. Auch eine lokale Applikation von Opioiden im Kniegelenk kann nach neuen Erkenntnissen dank peripherer Opioidrezeptoren deutlich schmerzhemmend wirken [16]. Aufgrund dieser Erfolge gelangt zunehmend auch die kontinuierliche intraartikuläre Infiltration von Lokalanästhetika und Opioiden mittels spezieller intraartikulärer Kathetersysteme (IAK) in den Fokus [10, 13, 20]. Aus einer elastomeren Ballonpumpe kann mittels dieser Technik die kontinuierliche Abgabe von Medikamenten in den ersten Tagen postoperativ direkt in das Kniegelenk erfolgen.

Ziel der Studie war es, mittels eines standardisierten postoperativen Behandlungsalgorithmus prospektiv eines dieser neuen IAK-Systeme mit der Applikation eines NFK über den Zeitraum der ersten 5 postoperativen Tage zu vergleichen im Hinblick auf:

- Schmerzintensität,

- Kniegelenksbeweglichkeit,

- Fähigkeit der selbstständigen Mobilisation,

- zusätzlichen Opioidbedarf,

- Einleitungs- bzw. Operationszeit,

- stationäre Aufenthaltsdauer.

\section{Methodik}

\section{Studiendesign}

Es handelt sich um eine monozentrische, prospektiv randomisierte, zweiarmige Parallelstudie. Diese wurde entsprechend unter dem Titel „Postoperative Analgesie nach Knie-TEP: Intraartikulärer Katheter vs. Nervus femoralis Katheter" im Deutschen Register Klinischer Studien (DRKS) in Freiburg unter der Registrierungsnummer DRKS 00006146 im Vorfeld der Untersuchung am 23.05.2014 registriert. Ein positives Ethikvotum der Ethikkommission der Landesärztekammer Hessen in Frankfurt vom 03.04.2014 lag vor Beginn des Patienteneinschlusses vor. Alle Untersuchungen wurden entsprechend der Deklaration von Helsinki durchgeführt.

\section{Studienpopulation}

Es wurden konsekutiv Patienten beider Geschlechter, im Alter von 50-85 Jahren, indiziert für eine primäre Knie-TEP aufgrund fortgeschrittener Gonarthrose eingeschlossen (• Tab. 1). Ausgeschlossen wurden Patienten zur simultanen bilateralen Versorgung oder bei Indikation zu einem unilateralen Oberflächenersatz. Weitere Ausschlusskriterien waren ASA IV (American Society of Anesthesiologists), eine orale Antikoagulation mittels Phenprocoumon, NOAK (neue orale Antikoagulanzien) oder Clopidogrel sowie eine präoperative langjährige Opiateinnahme aufgrund chronischer Schmerzen.
Patienten mit bekannten Allergien oder Unverträglichkeiten gegen Lokalanästhetika oder Opiate wurden ebenfalls ausgeschlossen.

\section{Patienteneinschluss und Randomisierung}

Die Patientenrekrutierung erfolgte von Juni 2014 bis November 2014. Potenzielle Studienteilnehmer wurden ausführlich über den Inhalt der Studie und mögliche Risiken aufgeklärt. Die Teilnahme erfolgte freiwillig. Eine schriftliche Einverständniserklärung wurde entsprechend eingeholt.

Die Randomisierung erfolgte im Anschluss durch das Ziehen eines aus einem gemischten Pool von insgesamt $120 \mathrm{Ku}$ verts (60 x Gruppe A, 60 x Gruppe B) durch eine unbeteiligte Person. Patienten, welche der Gruppe A zugeteilt wurden bekamen intraoperativ einen IAK gelegt (• Abb. 1), Patienten der Gruppe B erhielten im Rahmen der Narkoseeinleitung einen NFK ( $\bullet$ Abb. 2). Das mittlere Patientenalter betrug $70,5 \pm 8,3$ Jahre (5185 Jahre). Es wurden 77 (64,2\%) Frauen und $43(35,8 \%)$ Männer operiert, in 50 $(41,7 \%)$ Fällen war die linke Seite betroffen, in $70(58,3 \%)$ Fällen die rechte Seite.

\section{Untersuchungsablauf}

Die zu untersuchenden Therapieverfahren waren die Einlage eines IAK sowie die Applikation eines NFK. In Gruppe A erfolgte die Einlage des IAK (InfiltraLong, Fa. Pajunk ${ }^{\circledR}$, Geisingen; • Abb. 1) intra- 
Orthopäde 2015 - 44:566-573 DOI 10.1007/s00132-015-3115-y

(c) Die Autor(en) 2015. Dieser Artikel ist auf Springerlink.com mit Open Access verfügbar

K.P. Kutzner · C. Paulini · M. Hechtner · P. Rehbein · J. Pfeil

Postoperative Analgesie nach Knie-TEP. Intraartikulärer Katheter vs. Nervus-femoralis-Katheter

\section{Zusammenfassung}

Hintergrund. Das postoperative Schmerzmanagement nach Knie-TEP-Implantation stellt weiterhin eine große Herausforderung dar. Im Rahmen von neuen Fast-track-Konzepten nimmt die Bedeutung vor allem von multimodalen Therapien zu. Neue kontinuierliche intraartikuläre Infiltrationskathetersysteme (IAK) stehen im Hinblick auf eine effektive Schmerzbehandlung, Reduzierung von Nebenwirkungen sowie zur zügigen Mobilisation der Patienten auf dem Prüfstand.

Methodik. Insgesamt 120 Patienten wurden im Rahmen der Implantation einer Knie-TEP in eine prospektive randomisierte Vergleichsstudie eingeschlossen. In einem standardisierten Therapiealgorithmus bekamen $60 \mathrm{~Pa}$ tienten einen IAK (Gruppe A), während bei 60 Patienten ein Nervus-femoralis-Katheter (NFK) appliziert wurde (Gruppe B). Alle weiteren perioperativen Therapiebestandteile waren für alle Patienten identisch. In den ers- ten 5 Tagen postoperativ wurde die Schmerzintensität (VAS), passive und aktive Flexion, Opioidbedarf und die selbstständige bzw. Flurmobilisation untersucht und dokumentiert. Die Einleitungszeit, Schnitt-Naht-Zeit und die stationäre Aufenthaltsdauer wurden erfasst.

Ergebnisse. Bezüglich der Schmerzintensität konnten keine signifikanten Unterschiede gemessen werden (MW 5, $1 \pm 2,5$ vs. 4,6 $\pm 2,6$; $p=0,27)$. Ebenfalls zeigten sich hinsichtlich des zusätzlichen Opioidbedarfs sowie der Kniegelenksbeweglichkeit keine statistisch relevanten Vorteile einer Therapie. In der Gruppe der IAK konnte eine deutlich raschere selbstständige Mobilisation erreicht werden $(p<0,001)$. Die durchschnittliche Einleitungszeit unterschied sich deutlich im Vergleich zur NFK-Gruppe um 11,9 min $(p<0,001)$. Hinsichtlich der Schnitt-Naht-Zeit und der stationären Aufenthaltsdauer war kein Unterschied auffällig. Die Fehlerrate bzw. Dislokationsrate des NFK erscheint erhöht.

Diskussion. Die perioperative Behandlung mit einem IAK ist eine einfache Technik und hat im Vergleich zur Schmerztherapie mit einem NFK eine deutlich schnellere selbstständige Mobilisation zur Folge. Der Einsatz dieser Technik kann also empfohlen werden. Im Vergleich der Schmerzintensität, des zusätzlichen Bedarfes an Opioiden und der Kniegelenksbeweglichkeit bieten sich in dieser Untersuchung keine Vorteile im Vergleich zum NFK. Durch eine Zeitersparnis ist eine Kostenreduktion möglich.

\section{Schlüsselwörter}

Katheter · Knie-TEP · Nervenblock .

Opioidanalgesie $\cdot$ Randomisierte kontrollierte Studie

\section{Postoperative analgesia after total knee arthroplasty. Continuous intra-articular catheter vs. continuous femoral nerve block}

\section{Abstract}

Background. Postoperative pain management after total knee arthroplasty (TKA) remains a great challenge even today. In the context of new fast-track concepts, the importance of multimodal therapies for the effective treatment of pain and the reduction of side effects, as well as for the rapid ambulation of patients is increasing. Therefore, new continuous intra-articular catheter-systems (IAC) are under investigation.

Materials and methods. A total of 120 patients receiving total knee replacement were included in a prospective randomized comparative study. In a standardized treatment regime 60 patients received an IAC (group A), while in 60 patients a continuous femoral nerve block (FNB) was applied (group B). All other perioperative therapy components were identical for all patients. In the first 5 days after surgery pain intensity (VAS), passive and active flexion, opioid requirements, and self-initiated or hallway ambulation were investigated and documented. The initiation time, operation time, and length of hospital stay were recorded.

Results. Regarding pain intensity no significant differences occurred (mean $5.1 \pm 2.5$ vs. $4.6 \pm 2.6 ; p=0.27$ ). Also, additional opioid requirements and range of motion (ROM) showed no relevant distinctions of therapy. In the IAC group a much more rapid independent mobilization was achieved $(p<0.001)$. The mean initiation time before surgery decreased markedly compared to the FNB group by $11.9 \mathrm{~min}(p<0.001)$. With respect to the operation time and duration of hospital stay there was no noticeable difference. The failure rate and the rate of dislocation of FNB appear to be increased.

Conclusion. The perioperative treatment with an IAC system is an easy technique, which ensures a markedly faster ambulation following TKA compared to the treatment with continuous FNB. Hence, its usage, especially in fast-track concepts can be recommended. In this study, a comparison of pain intensity, the additional requirement of opioids and early range of motion (ROM) offers no benefits compared to FNB. Due to time savings cost reduction can be achieved.

\section{Keywords}

Catheter · Nerve block · Opioid analgesics . Randomized controlled trial · Total knee replacement operativ über eine Spaltverweilkanüle unmittelbar vor dem Faszienverschluss. An den Katheter wurde eine elastomere Infusionspumpe (FuserPump - $350 \mathrm{ml}$, Fa. Pajunk $^{\circledR}$, Geisingen; - Abb. 1) angeschlossen, welche ein Gemisch aus $200 \mathrm{ml}$ Ropivacain $(7,5 \mathrm{mg} / \mathrm{ml}$; Fa. AstraZeneca, London), 2 ml Morphin (10 mg/ml; Fa. Merck,
Darmstadt) und $148 \mathrm{ml} \mathrm{NaCl}$ (0,9\%; Fa. B. Braun, Melsungen) enthält und eine kontinuierliche Infusionsrate von $8 \mathrm{ml} / \mathrm{h}$ für insgesamt etwa $44 \mathrm{~h}$ gewährleistet. Der IAK wurde nach Aufbrauchen des Gemisches entfernt. Gruppe B erhielt im Rahmen der Operationseinleitung einen NFK (PlexoLong/Meier, Fa. Pajunk ${ }^{\circledR}$, Geisin- gen; Abb. 2) mit entsprechender Pumpe (Pega ${ }^{\circledR}$ Plus, Fa. Venner Medical, Dänischenhagen), welche eine kontinuierliche Flussrate von $8 \mathrm{ml} / \mathrm{h}$ Ropivacain (2 mg/ $\mathrm{ml}$ ) gewährleistet. Die Entfernung erfolgte am Morgen des 3. postoperativen Tages.

Alle weiteren perioperativen Therapiebestandteile, wie Intubationsnarkose, 


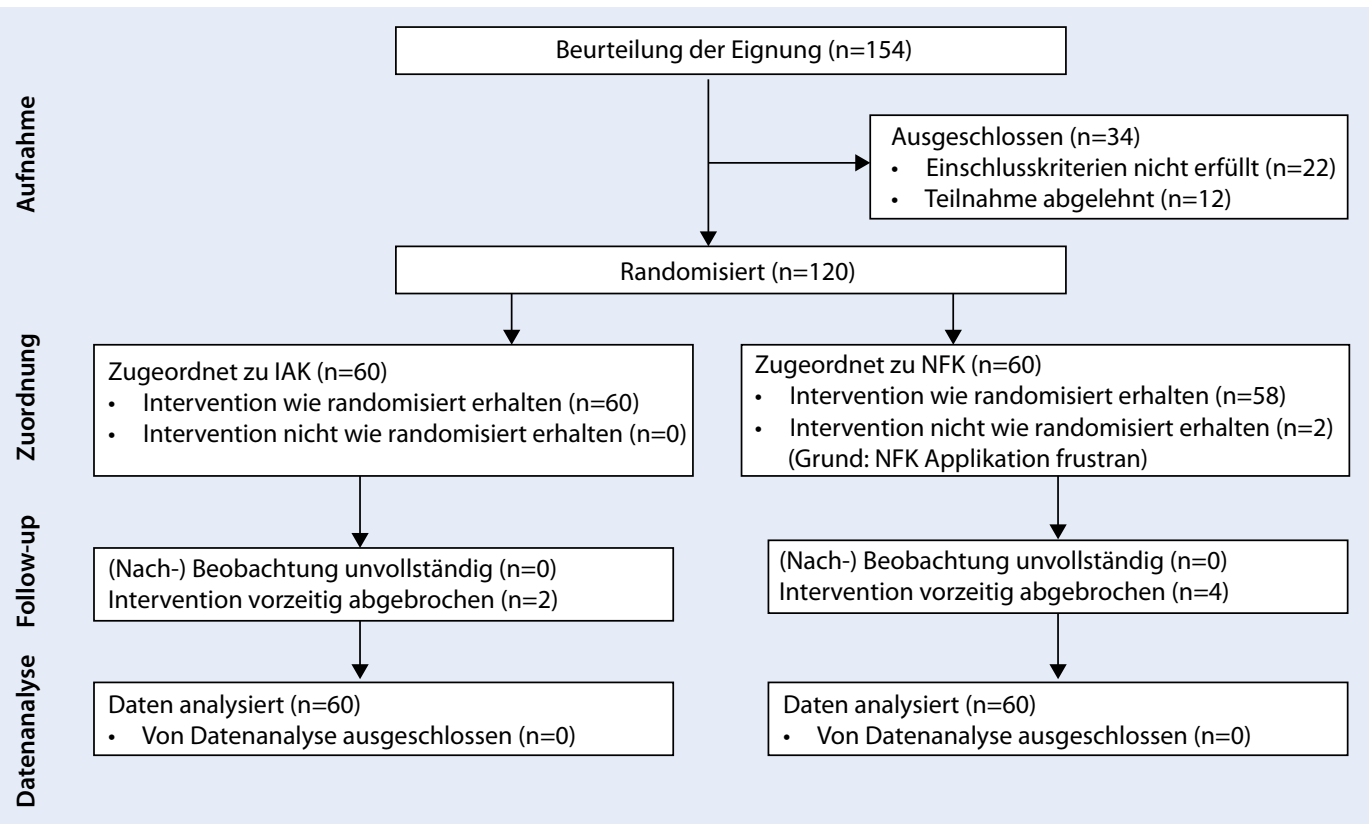

Abb. $3<$ Flussdiagramm der ein- und ausgeschlossenen Teilnehmer im Verlauf der Studie (IAK intraartikuläres Kathetersystem, NFK Nervus-femoralis-Katheter)

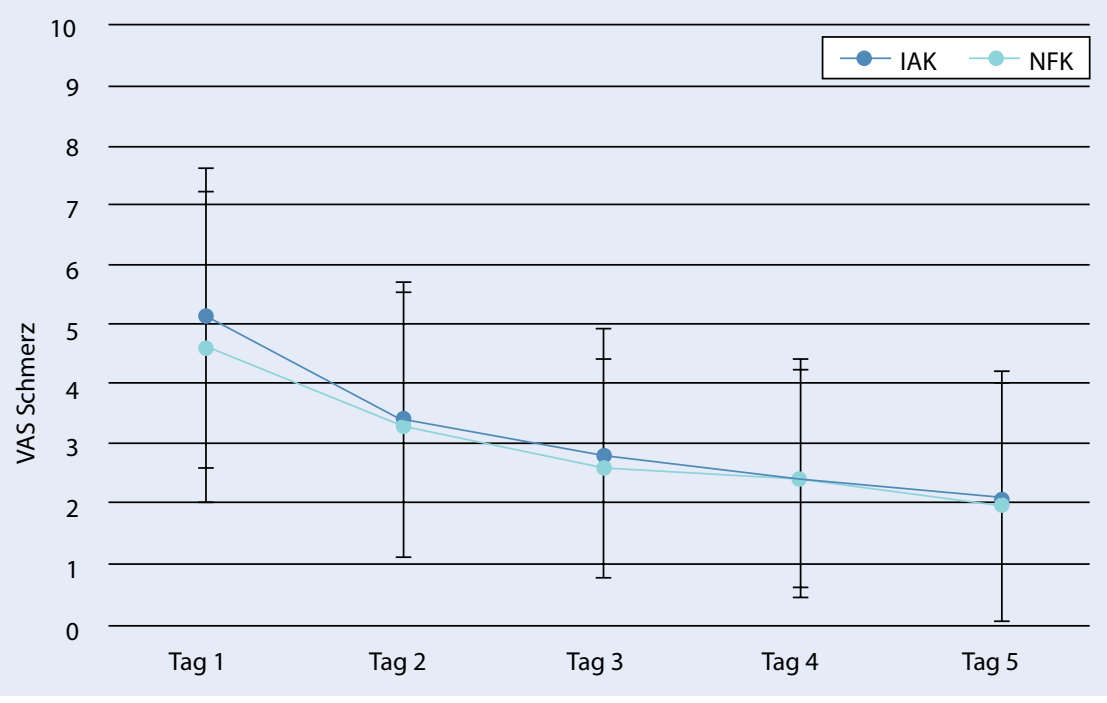

Abb. $4 \Delta$ Schmerzintensität nach VAS Schmerz (Visuellen Analog Skala) an Tag 1-5 postoperativ (IAK intraartikuläres Kathetersystem, NFK Nervus-femoralis-Katheter)

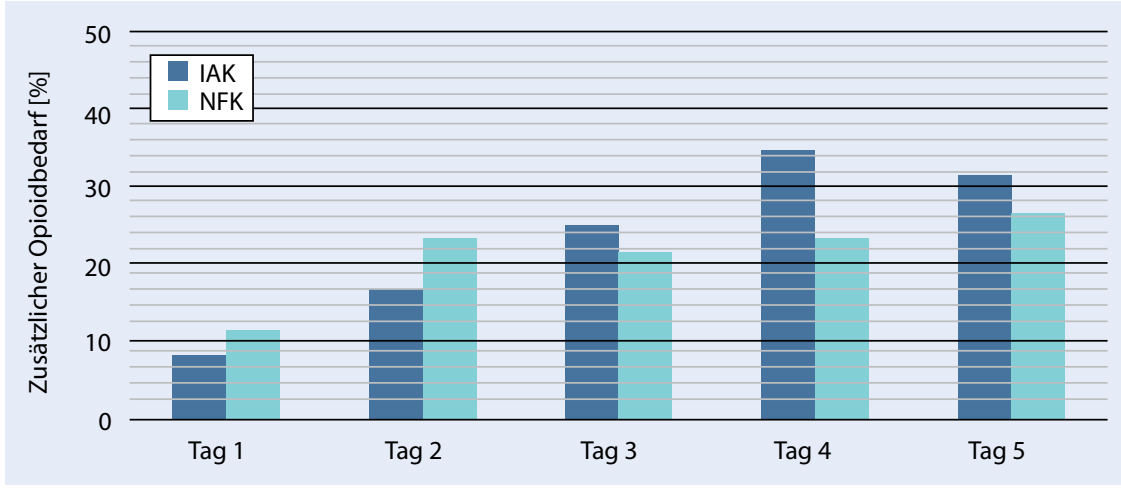

Abb. $5 \Delta$ Zusätzlicher Opioidbedarf an Tag 1-5 postoperativ (IAK intraartikuläres Kathetersystem, NFK Nervus-femoralis-Katheter)

Operationstechnik, Implantate, intraoperative Infiltration, Infektprophylaxe mittels Single-shot-Antibiotika, medikamentöse Thromboseprophylaxe und AVPumpe, orale Schmerzmedikation sowie physiotherapeutische Behandlung inklusive CPM-Schiene (continuous passive motion) waren für alle Patienten identisch. Die Nachbehandlung aller Patienten erfolgte in gleicher Weise. Sowohl das Pflege- als auch das physiotherapeutische Personal wurde zwecks identischer postoperativer Behandlung, auch im Sinne einer frühen Mobilisation ab dem 1. postoperativen Tag in beiden Gruppen, instruiert und kontrolliert. Alle Patienten dokumentierten postoperativ täglich zu einem standardisierten Zeitpunkt nach dem Frühstück die Schmerzintensität entsprechend der Visuellen Analog Skala (VAS 0-10) Schmerz. Zusätzlich wurden in einer täglichen zusätzlichen Visite die passive bzw. aktive Flexion, der Opioidkonsum, das selbstständige Aufstehen und die Flurmobilisation untersucht und dokumentiert. Retrospektiv wurden Einleitungszeit, Schnitt-Naht-Zeit und die stationäre Aufenthaltsdauer erhoben.

\section{Operation}

Alle Patienten bekamen eine Intubationsnarkose. Die Eingriffe wurden von erfahrenen Operateuren in standardisierter Weise durchgeführt. Eine Blutleere mit 


\section{Originalien}

\begin{tabular}{|c|c|c|c|c|c|c|}
\hline & \multicolumn{2}{|l|}{ IAK } & \multicolumn{2}{|l|}{ NFK } & \multicolumn{2}{|c|}{ Gesamt } \\
\hline & MW & SD & MW & SD & MW & SD \\
\hline Tag 1 & 5,1 & $\pm 2,5$ & 4,6 & $\pm 2,6$ & 4,8 & $\pm 2,5$ \\
\hline Tag 2 & 3,4 & $\pm 2,3$ & 3,3 & $\pm 2,2$ & 3,4 & $\pm 2,3$ \\
\hline Tag 3 & 2,8 & $\pm 2,1$ & 2,6 & $\pm 1,8$ & 2,7 & $\pm 2,0$ \\
\hline Tag 4 & 2,4 & $\pm 2,0$ & 2,4 & $\pm 1,8$ & 2,4 & $\pm 1,9$ \\
\hline Tag 5 & 2,1 & $\pm 2,1$ & 2 & $\pm 2,0$ & 2 & $\pm 2,0$ \\
\hline
\end{tabular}

IAK intraartikuläres Kathetersystem, MW Mittelwert, NFK Nervus-femoralis-Katheter, SD Standardabweichung

\begin{tabular}{|c|c|c|c|c|c|c|}
\hline & IAK & & NFK & & Gesamt & \\
\hline & Passiv & Aktiv & Passiv & Aktiv & Passiv & Aktiv \\
\hline & $M W \pm S D$ & & MW & SD & MW & SD \\
\hline Tag 1 & $50,9 \pm 13,8$ & $32,6 \pm 13,2$ & $57,4 \pm 20,6$ & $34,0 \pm 18,0$ & $54,2 \pm 17,8$ & $33,3 \pm 15,7$ \\
\hline Tag 2 & $60,5 \pm 16,2$ & $43,1 \pm 14,4$ & $70,4 \pm 16,6$ & $48,0 \pm 19,1$ & $65,5 \pm 17,1$ & $45,5 \pm 17,0$ \\
\hline $\operatorname{Tag} 3$ & $72,8 \pm 14,3$ & $55,6 \pm 16,6$ & $79,4 \pm 12,9$ & $62,9 \pm 16,8$ & $76,1 \pm 13,9$ & $59,3 \pm 17,0$ \\
\hline Tag 4 & $81,3 \pm 10,7$ & $67,6 \pm 14,9$ & $85,4 \pm 10,2$ & $72,0 \pm 16,9$ & $83,3 \pm 10,6$ & $69,8 \pm 16,0$ \\
\hline Tag 5 & $87,9 \pm 7,3$ & $73,5 \pm 15,7$ & $90,6 \pm 9,3$ & $78,5 \pm 13,9$ & $89,3 \pm 8,4$ & $76,0 \pm 15,0$ \\
\hline $\begin{array}{l}\text { Maximal } \\
\text { (in 5 Tagen) }\end{array}$ & $88,6 \pm 7,1$ & $76,4 \pm 12,0$ & $91,2 \pm 9,1$ & $79,4 \pm 14,0$ & $89,9 \pm 8,2$ & $77,9 \pm 13,1$ \\
\hline
\end{tabular}

$350 \mathrm{mmHg}$ wurde angelegt. Alle Eingriffe erfolgten über den medialen parapatellaren Zugang. Alle Patienten wurden mit dem zementierten, bikondylären Oberflächenersatz Genesis II PS (Fa. Smith and Nephew, London) versorgt. Es erfolgte regelhaft die Einlage einer subfaszialen Redon-Drainage. Diese wurde postoperativ zur Anflutung eine halbe Stunde verschlossen gehalten, anschließend auf Sog eingestellt und regelhaft am 2 . postoperativen Tag entfernt.

Vor dem Wundverschluss erfolgte bei allen Patienten eine periartikuläre Infiltration der Kapsel, des Retinakulums und des subkutanen Weichteilgewebes mit einem Gemisch aus $15 \mathrm{ml}$ Ropivacain $(7,5 \mathrm{mg} / \mathrm{ml})$ und $10 \mathrm{ml} \mathrm{NaCl}(0,9 \%)$. Nach dem Wundverschluss erfolgte die Gabe des gleichen Gemisches $(25 \mathrm{ml})$ als Bolus intraartikulär über die Redon-Drainage.

\section{Postoperative Basismedikation}

In beiden Behandlungsgruppen wurde eine postoperative Basismedikation bestehend aus dem NSAR Etoricoxib (1×90 mg; Fa. Grünenthal, Aachen) und Novaminsulfon $(4 \times 500 \mathrm{mg}$; Fa. Ratiopharm, Ulm) verabreicht. Bei Unverträglichkeit wurde das entsprechende Medikament pausiert. Im Bedarfsfall er- folgte die Gabe von Paracetamol intravenös ( $1 \mathrm{~g} ; 10 \mathrm{mg} / \mathrm{ml}$; Fa. B. Braun, Melsungen).

Bei nicht ausreichender Wirksamkeit bzw. anhaltender Schmerzangabe durch den Patienten erfolgte die Gabe des oralen Opioids Oxycodon-Hydrochlorid $(2 \times 10 \mathrm{mg} ; \mathrm{Fa}$. Mundipharma, Limburg $)$ oder Piritramid intramuskulär bei Bedarf (7,5 mg/ml; Fa. Janssen, Neuss). Die Notwendigkeit der zusätzlichen Einnahme eines Opioids wurde täglich entsprechend dokumentiert und ausgewertet.

\section{Statistik}

Primärer Endpunkt der Studie war die Schmerzintensität auf der VAS Schmerz an Tag 1. Als sekundäre Endpunkte wurden die Schmerzintensität auf der VAS Schmerz Tag 2-5, passive und aktive Flexion, zusätzlicher Opioidbedarf, selbstständige bzw. Flurmobilisation, Einleitungszeit, Schnitt-Naht-Zeit und stationäre Aufenthaltsdauer definiert. Vor Patienteneinschluss wurde eine Fallzahlplanung durchgeführt. Es erfolgten deskriptive Analysen aller Daten. Kategoriale Variablen wurden mittels absoluten und relativen Häufigkeiten, stetige Variablen durch Mittelwert und Standardabweichung (falls nicht normalverteilt durch Median und Interquartilsabstand) beschrieben. Der Therapievergleich hinsichtlich des primären Endpunkts wurde konfirmatorisch, alle weiteren Analysen explorativ durchgeführt. Das Signifikanzniveau wurde bei $\alpha=0,05$ festgelegt. Gruppenvergleiche für stetige Endpunkte wurden mittels t-test untersucht. Im Falle einer Nichtnormalverteilung mittels Mann-Whitney-U-Test. Bei kategorialen Endpunkten wurden Häufigkeitsvergleiche zwischen den Therapiegruppen mittels Chiquadrat-Test angestellt. Alle Analysen erfolgten nach dem Intention-totreat-Prinzip und wurden mit SPSS Version 22.0 (SPSS Inc., Chicago Illinois) durchgeführt.

\section{Ergebnisse}

Von insgesamt 154 möglichen Patienten im Studienzeitraum konnten 120 Patienten in die Studie eingeschlossen werden. - Abb. 3 zeigt das entsprechende Flussdiagramm. In 2 Fällen von eingeschlossenen Patienten war aufgrund eines adipösen Habitus das Legen eines NFK erfolglos. Diese Patienten erhielten keine Alternativtherapie. Bei 4 Patienten musste der NFK aufgrund von Insuffizienz bei Dislokation vorzeitig entfernt werden. In der Gruppe der IAK kam es zu 2 vorzeitigen Dislokationen. Es zeigte sich bei einem Patienten ein oberflächlicher Infekt im Bereich der NFK-Einstichstelle in der Leiste, welcher im Verlauf des stationären Aufenthalts mit lokaler und antibiotischer Therapie zur Ausheilung gebracht werden konnte. Bei Verdacht auf Frühinfekt erfolgte bei einem weiteren Patienten in der NFK-Gruppe eine einmalige Wundrevision. Eine Infektion bestätigte sich nicht. Es zeigten sich insgesamt keine Sturzereignisse. Ebenfalls zeigten sich keine tiefen Beinvenenthrombosen.

\section{Primärer Endpunkt}

Die mittels VAS Schmerz gemessenen Schmerzintensitäten am 1. postoperativen Tag zeigten keine signifikanten Unterschiede im Vergleich der beiden Gruppen. Der mittlere VAS-Wert an Tag 1 betrug $5,1 \pm 2,5$ (IAK) vs. $4,6 \pm 2,6$ (NFK) $(p=0,27)(\bullet$ Abb. 4; $\bullet$ Tab. 2). 


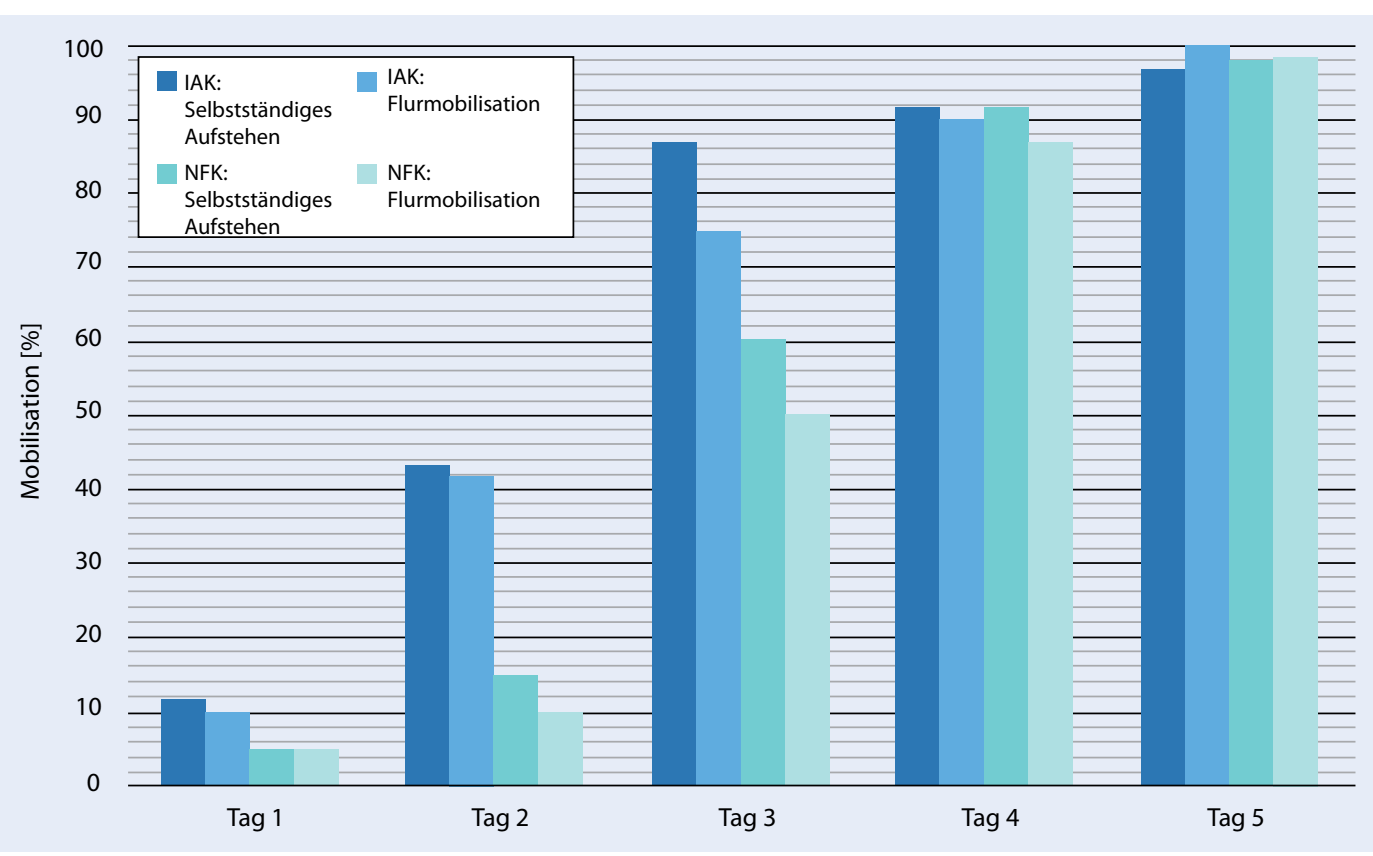

Abb. $6<$ Mobilisation an Tag 1-5 postoperativ: Selbstständiges Aufstehen und Flurmobilisation an Unterarmgehstützen (IAK intraartikuläres Kathetersystem, NFK Nervus-femoralis-Katheter)

\section{Sekundäre Endpunkte}

Auch im weiteren zeitlichen Verlauf an Tag 2-5 waren keine statistisch auffälligen Unterschiede erkennbar ( $\bullet$ Abb. 4;

- Tab. 2). A Abb. 2 zeigt den zeitlichen Verlauf über 5 Tage. Auch im Hinblick auf die passive- und aktive Kniegelenksflexion ergaben sich keine statistisch auffälligen Unterschiede (• Tab. 3). Die mittlere maximale passive Flexion in den ersten 5 Tagen betrug in Gruppe A $88,6 \pm 7,1^{\circ}$ und in Gruppe B $91,2 \pm 9,1^{\circ}$ $(p=0,09)$. In Gruppe A erreichten $45 \mathrm{~Pa}$ tienten $(75,0 \%)$ innerhalb der ersten $5 \mathrm{Ta}$ ge eine passive Flexion von $90^{\circ}$. In Gruppe $B$ wurde dies von 46 Patienten $(76,7 \%)$ erreicht $(p=0,83)$. Der durchschnittliche Bedarf an zusätzlichen Opioiden ergab ebenfalls keine relevanten Unterschiede (• Abb. 5). 23 (38,3\%) Patienten in Gruppe A und 21 (35,0\%) Patienten in Gruppe B nahmen an mindestens einem Tag zusätzliche Opioide ein $(p=0,71)$.

- Abb. 5 zeigt den zeitlichen Verlauf. Das selbstständige Aufstehen sowie die Mobilisation auf dem Stationsflur gelangen Patienten der Gruppe A deutlich rascher. Beispielsweise waren 25 (41,7\%) aus Gruppe A am 2. Tag bereits mit Gehstützen auf dem Flur mobilisiert, während dies nur $6(10,2 \%)$ aus Gruppe B schafften $(p<0,001)$ ( Abb. 6). Die mittlere Einleitungszeit betrug in Gruppe A
$42,8 \pm 10,0 \mathrm{~min}, 54,7 \pm 9,8 \mathrm{~min}$ in Gruppe B $(p<0,001)$. Die Schnitt-Naht-Zeit betrug $75,3 \pm 21,1 \mathrm{~min}$ in Gruppe A und $81,0 \pm 23,9$ min in Gruppe B $(p=0,17)$. Keine statistisch auffälligen Unterschiede zeigten sich bzgl. der stationären Aufenthaltsdauer (• Tab. 1).

\section{Diskussion}

In der vorliegenden Studie zeigte sich hinsichtlich der Schmerzintensität in den ersten Tagen postoperativ kein Vorteil der kontinuierlichen intraartikulären Infiltration mittels intraartikulärem Kathetersystem im Vergleich zur Therapie mittels Nervus-femoralis-Katheter. Die Kniegelenksfunktion stellte sich ebenfalls gleich dar. In der Gruppe der IAK konnte jedoch eine signifikant raschere Mobilisation der Patienten erfolgen, das selbstständige Aufstehen und die Flurmobilisation wurden zu einem früheren Zeitpunkt erreicht.

In den letzten Jahren konnte bereits eine Wirksamkeit einer Behandlung mittels IAK gezeigt werden. Goyal et al. randomisierten 2013 insgesamt 150 Patienten nach Knie-TEP in Gruppen mit Bupivacain oder $\mathrm{NaCl}$ als Kontrolle. Verringerte Schmerzintensität und reduzierter Opioidbedarf waren die Folge [14]. Siebert berichtete 2013 über erste Erfahrungen in der Anwendung von intraartikulärem Ropivacain nach Knie-TEP in 1252
Fällen [24]. Eine gute Reduzierung der postoperativen Schmerzen, eine Einsparung von Opioiden und eine rasch mögliche Mobilisation der Patienten waren das Ergebnis. Tsukada et al. verglichen 2014 eine Gruppe mit Epiduralanalgesie mit einer Gruppe mit einer einmaligen perioperativen intraartikulären Injektion. Die Ergebnisse waren eine reduzierte Schmerzsymptomatik und geringere Nebenwirkungen [25].

Ein Wirksamkeitsnachweis in einem prospektiv randomisierten Vergleich mit der weitverbreiteten Methode des NFK liegt jedoch bis zum heutigen Tage nicht vor. 2014 konnten Reinhardt et al. ebenfalls keine Schmerzreduktion im Vergleich einer kontinuierlichen intraartikulären Analgesie mit einer Epiduralanalgesie plus einmaligem Nervus-femoralisBlock nachweisen. Jedoch kam es in der Epiduralgruppe zu einer deutlich verzögerten Mobilisation. Dies deckt sich mit den Ergebnissen der vorliegenden Studie.

Der Zeitunterschied im Rahmen der OP-Einleitung ist auf das technisch aufwendige Legen eines NFK zurückzuführen, während die Applikation des IAK intraoperativ im Rahmen der Zementaushärtung erfolgen kann und somit keinen Zeitverlust bedeutet.

Der fehlende Unterschied im Bereich der stationären Aufenthaltsdauer ist als Folge einer zunehmend unflexiblen Poli- 
tik vieler Kostenträger zu werten, wodurch ein Entlassungstermin in eine stationäre Rehabilitationseinrichtung zeitlich zum Teil deutlich verzögert wird. Im Rahmen von Fast-track-Konzepten gilt es hier neue Wege zu gehen.

\section{Limitationen}

Die vorliegende Studie beinhaltet Limitationen. Zum einen waren Patienten und behandelndes Personal nicht verblindet. Dies hätte einen deutlich höheren Aufwand bedeutet, denn auch das gesamte Pflege- und physiotherapeutische Personal hätte integriert werden müssen. Eine dadurch resultierende Differenz zwischen den beiden Behandlungsgruppen, vor allem in Bezug auf eine frühe Mobilisation, ist denkbar. Die Therapieregime wurden jedoch explizit für beide Gruppen in gleicher Weise standardisiert, das Personal entsprechend geschult und während der Studiendauer kontrolliert. Des Weiteren ist der Beobachtungszeitpunkt sehr kurz gewählt und Aussagen über mittel- und langfristige Ergebnisse können daraus nicht abgeleitet werden. Jedoch war dies auch nicht Gegenstand der Studie.

Die für die Fallzahlplanung angenommene Effektgröße und Standardabweichung wichen von den tatsächlich beobachteten Werten geringfügig $\mathrm{ab}$, wodurch sich die statistische Aussagekraft der Analysen leicht reduzierte. Es handelt sich bei der vorliegenden Studie dennoch um die derzeit größte Untersuchung zu diesem Therapievergleich, welche eine gute $\mathrm{Ge}$ neralisierbarkeit der Ergebnisse gewährleistet.

\section{Ausblick}

Die Ergebnisse dieser Vergleichsstudie legen einen zunehmenden Einsatz von intraartikulären Infiltrationen mittels IAK nach Knie-TEP-Implantation nahe. Gerade im Rahmen von Fast-track-Konzepten ist eine schnelle und sichere Mobilisation entscheidend. Auch im Hinblick auf die zunehmende Ökonomisierung der Kliniken mit notwendiger Kostenreduktion werden kürzere OP-Vorbereitungszeiten von Vorteil sein. In Zukunft sollte untersucht werden, inwieweit die Fähigkeit der rascheren Mobilisation der Patienten eine kürzere stationäre Aufenthaltsdauer möglich macht. Ein zunehmendes Umdenken in Richtung ambulanter Rehabilitationsmaßnahmen und damit flexiblerer Entlassungsmöglichkeiten erscheint hierbei sinnvoll. Zukünftige Untersuchungen sollten jedoch weiterhin kritisch eine adäquate Schmerztherapie adressieren.

\section{Fazit für die Praxis}

- Die perioperative Behandlung mittels IAK erlaubt eine sichere und raschere, selbstständige Mobilisation.

- Hinsichtlich Schmerzintensität und Kniegelenksbeweglichkeit bietet der IAK im Vergleich zum NFK in den ersten Tagen keine Vorteile. Die klinischen Ergebnisse sind in beiden Gruppen nach 5 Tagen ausgezeichnet.

- Beide Techniken sind wirkungsvolle und nebenwirkungsarme Methoden, welche im Rahmen orthopädischer Operationen angeboten werden sollten.

- Ein verstärktes Umdenken in Richtung ambulanter Rehabilitationsmaßnahmen aufgrund zeitintensiver, bürokratischer Prozesse im Bereich der Kostenträger erscheint wünschenswert.

Kr. K.P. Kutzner
Klinik für Orthopädie und
Unfallchirurgie
St. Josefs Hospital Wiesbaden
Beethovenstr. 20
65189 Wiesbaden
kkutzner@joho.de

\section{Einhaltung ethischer Richtlinien}

Interessenkonflikt. K.P. Kutzner, C. Paulini, M. Hechtner, P. Rehbein, J. Pfeil geben an, dass kein Interessenskonflikt besteht.

Alle beschriebenen Untersuchungen am Menschen wurden mit Zustimmung der zuständigen Ethik-Kommission, im Einklang mit nationalem Recht sowie gemäß der Deklaration von Helsinki von 1975 (in der aktuellen, überarbeiteten Fassung) durchgeführt. Von allen beteiligten Patienten liegt eine Einverständniserklärung vor.
Open Access. Dieser Artikel unterliegt den Bedingungen der Creative Commons Attribution License. Dadurch sind die Nutzung, Verteilung und Reproduktion erlaubt, sofern der/die Originalautor/ en und die Quelle angegeben sind.

\section{Literatur}

1. American Society of Anaesthesiologists Task Force on Acute Pain Management (2004) Practice guidelines for acute pain management in the perioperative setting: an updated report by the American Society of Anesthesiologists Task Force on Acute Pain Management. Anesthesiology 100:15731581

2. Beaussier M (1998) [Frequency, intensity, development and repercussions of postoperative pain as a function of the type of surgery]. Ann Fr Anesth Reanim 17:471-493

3. Brasseur L, Boukhatem B (1998) [The epideiology of postoperative pain]. Ann Fr Anesth Reanim 17:534-539

4. Brokelman RBG, van Loon CJM, Rijnberg WJ (2003) Patient versus surgeon satisfaction after total hip arthroplasty. J Bone Joint Surg Br 85:495-498

5. Burroughs TE, Davies AR, Cira JC, Dunagan WC (1999) Understanding patient willingness to recommend and return: a strategy for prioritizing improvement opportunities. Jt Comm J Qual Improv 25:271-287

6. Busch CA, Shore BJ, Bhandari R et al (2006) Efficacy of periarticular multimodal drug injection in total knee arthroplasty. A randomized trial. J Bone Joint Surg Am 88:959-963. doi:10.2106/JBJS.E.00344

7. Chelly JE, Ben-David B, Williams BA, Kentor ML (2003) Anesthesia and postoperative analgesia: outcomes following orthopedic surgery. Orthopedics 26:865-871

8. Dalury DF, Lieberman JR, MacDonald SJ (2011) Current and innovative pain management techniques in total knee arthroplasty. J Bone Joint Surg Am 93:1938-1943. doi:10.2106/JBJS.9320icl

9. DeLeo JA (2006) Basic science of pain. J Bone Joint Surg Am 88(Suppl 2):58-62. doi:10.2106/ JBJS.E.01286

10. Dobrydnjov I, Anderberg C, Olsson C et al (2011) Intraarticular vs. extraarticular ropivacaine infusion following high-dose local infiltration analgesia after total knee arthroplasty: a randomized doubleblind study. Acta Orthop 82:692-698. doi:10.3109/ 17453674.2011.625535

11. Duarte VM, Fallis WM, Slonowsky D et al (2006) Effectiveness of femoral nerve blockade for pain control after total knee arthroplasty. J Perianesth Nurs 21:311-316. doi:10.1016/j.jopan.2006.05.011

12. Frassanito L, Vergari A, Zanghi F et al (2010) Postoperative analgesia following total knee arthroplasty: comparison of low-dose intrathecal morphine and single-shot ultrasound-guided femoral nerve block: a randomized, single blinded, controlled study. Eur Rev Med Pharmacol Sci 14:589-596

13. Gómez-Cardero P, Rodríguez-Merchán EC (2010) Postoperative analgesia in TKA: ropivacaine continuous intraarticular infusion. Clin Orthop Relat Res 468:1242-1247. doi:10.1007/s11999-009-1202-2

14. Goyal N, McKenzie J, Sharkey PF et al (2013) The 2012 Chitranjan Ranawat award: intraarticular analgesia after TKA reduces pain: a randomized, double-blinded, placebo-controlled, prospective study. Clin Orthop Relat Res 471:64-75. doi:10.1007/s11999-012-2596-9 
15. Hing CB, Stiehl JB (2013) Pain control following total knee replacement surgery. Knee 20:299. doi:10.1016/j.knee.2013.08.001

16. Jagla C, Martus P, Stein C (2014) Peripheral opioid receptor blockade increases postoperative morphine demands-A randomized, double-blind, placebo-controlled trial. Pain 155:2056-2062. doi:10.1016/j.pain.2014.07.011

17. Joo J-H, Park J-W, Kim J-S, Kim Y-H (2011) Is intra-articular multimodal drug injection effective in pain management after total knee arthroplasty? A randomized, double-blinded, prospective study. J Arthroplasty 26:1095-1099. doi:10.1016/j. arth.2011.03.052

18. Maheshwari A V, Blum YC, Shekhar L et al (2009) Multimodal pain management after total hip and knee arthroplasty at the Ranawat Orthopaedic Center. Clin Orthop Relat Res 467:1418-1423. doi:10.1007/s11999-009-0728-7

19. Paul JE, Arya A, Hurlburt L et al (2010) Femoral nerve block improves analgesia outcomes after total knee arthroplasty: a meta-analysis of randomized controlled trials. Anesthesiology 113:1144-1162. doi:10.1097/ALN.0b013e3181f4b18

20. Reeves M, Skinner MW (2009) Continuous intra-articular infusion of ropivacaine after unilateral total knee arthroplasty. Anaesth Intensive Care 37:918922

21. Reinhardt KR, Duggal S, Umunna B-P et al (2014) Intraarticular analgesia versus epidural plus femoral nerve block after TKA: a randomized, doubleblind trial. Clin Orthop Relat Res 472:1400-1408. doi:10.1007/s11999-013-3351-6

22. Rodgers A, Walker N, Schug S et al (2000) Reduction of postoperative mortality and morbidity with epidural or spinal anaesthesia: results from overview of randomised trials. BMJ 321:1493

23. Seet E, Leong WL, Yeo ASN, Fook-Chong S (2006) Effectiveness of 3-in-1 continuous femoral block of differing concentrations compared to patient controlled intravenous morphine for post total knee arthroplasty analgesia and knee rehabilitation. Anaesth Intensive Care 34:25-30

24. Siebert W (2013) Erste Erfahrungen in der Anwendung von intraartikulärem Ropivacain nach Knietotalendoprothese in 1252 konsekutiven Fällen. OUP 10:452-457. doi:10.3238/oup.2013.0452-0457

25. Tsukada S, Wakui M, Hoshino A (2014) Postoperative epidural analgesia compared with intraoperative periarticular injection for pain control following total knee arthroplasty under spinal anesthesia: a randomized controlled trial. J Bone Joint Surg Am 96:1433-1438. doi:10.2106/JBJS.M.01098

26. Wall PD (1988) The prevention of postoperative pain. Pain 33:289-290

\section{Kindesmisshandlung}

Jedes Jahr werden in Deutschland über

200.000 Kinder körperlich und seelisch misshandelt. Jede Wochen sterben 3 Kinder an

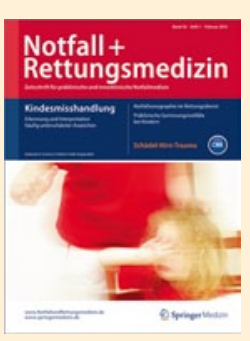
den Folgen körperlicher Gewalt. Diese Gewalt geschieht in jedem Milieu, ob Plattenbau, Reihenhaus oder Villa. Die derzeitige Zahl und Schwere der Kindesmisshandlun-

gen ist inakzeptabel und ihre Verringerung muss dringend als gesamtgesellschaftliche Aufgabe wahrgenommen werden. Besonders wichtig dabei ist es erste Anzeichen von Gewalt gegen Kinder im Alltag, in Praxis und Klinik zu erkennen und entsprechend zu handeln.

Lesen Sie im Schwerpunktheft „Kindesmisshandlung" (Ausgabe 1/2015) der Notfall+Rettungsmedizin wann ein Verdacht gerechtfertigt ist und wie Sie damit umgehen:

- Verdacht auf Kindesmisshandlung

- Diagnostische Kriterien der Hautverletzungen bei Kindesmisshandlung

- Tödliches Schütteltrauma

- Das Recht zur Weitergabe von Informationen bei Gefährdung des Kindeswohls

Bestellen Sie diese Ausgabe zum Preis von 38,- EUR zzgl. Versandkosten bei Springer Customer Service Center Kundenservice Zeitschriften Haberstr. 7

69126 Heidelberg

Tel.: +49 6221-345-4303

Fax: +49 6221-345-4229

E-Mail: leserservice@springer.com

Suchen Sie noch mehr zum Thema? Mit e.Med, dem Online-Paket von Springer Medizin, können Sie schnell und komfortabel in über 500 medizinischen Fachzeitschriften recherchieren.

Weitere Infos unter springermedizin.de/ eMed.

\section{Sicherheit von Medizinprodukten - Neueste Entwicklungen}

Medizinprodukte leisten einen wichtigen Beitrag zur Prävention, Diagnose und Therapie von Krankheiten. Gleichzeitig stehen

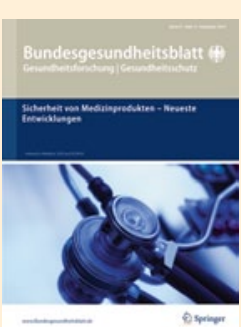

Medizinprodukte in einem Spannungsfeld: Forschung und Entwicklung schreiten schnell voran, minimalinvasive Operationsverfahren ermöglichen schonendere

Interventionen, intelligente Implantate unterstützen Körperfunktionen und bieten neue, medizinisch vielversprechende Behandlungsmöglichkeiten.

In der Zeitschrift Bundesgesundheitsblatt erschien der Schwerpunkt „Sicherheit von Medizinprodukten - Neueste Entwicklungen" (Ausgabe 12/2014) mit den folgenden Beiträgen zu den wichtigsten Aspekten des Themas:

- Medizinprodukte

- Erfahrungen und Empfehlungen des BfArM zur Genehmigung klinischer Prüfungen von Medizinprodukten und Bewertung schwerwiegender unerwünschter Ereignisse (SAE)

- Register als Werkzeug für mehr Endoprothesensicherheit

- Zukunftstrend „Medical Apps“

Bestellen Sie diese Ausgabe zum Preis von 16,- EUR zzgl. Versandkosten bei Springer Customer Service Center Kundenservice Zeitschriften

Haberstr. 7

69126 Heidelberg

Tel.: +49 6221-345-4303

Fax: +49 6221-345-4229

E-Mail: leserservice@springer.com

Suchen Sie noch mehr zum Thema? Mit e.Med, dem Online-Paket von Springer Medizin, können Sie schnell und komfortabel in über 500 medizinischen Fachzeitschriften recherchieren.

Weitere Infos unter springermedizin.de/ eMed. 